\section{Andrzej Persidok}

Facultad de Teología de la Universidad de Navarra e-mail: apersidok@alumni.unav.es

DOI: http://dx.doi.org/10.12775/BPTh.2015.018

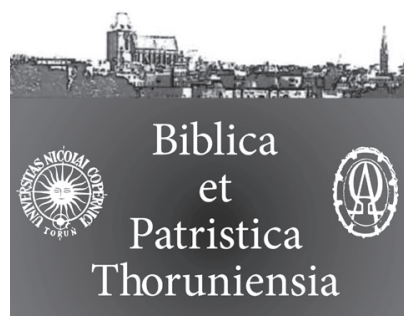

$8(2015) 3$

ISSN (print) 1689-5150

ISSN (online) 2450-7059

\title{
¿Revolucionario o «genio simplificador»? Santo Tomás de Aquino en la Exégèse médiévale de Henri de Lubac
}

\section{Revolutionary or «genius of simplification»? St. Thomas of Aquinas in Henri de Lubac's Exégèse médiévale}

Resumen. En el artículo se analiza la manera de que Henri de Lubac representó a santo Tomás de Aquino en su obra sobre la exégesis medieval. En la primera parte, se ofrece un estudio general de la obra. La segunda parte es un análisis del capítulo de la Exégèse médiévale dedicado a santo Tomás y a la edad escolástica. El estudio general de la obra previamente realizado permite comprender la manera específica de que el teólogo francés trata al Aquinate en caunto exégeta: sin fijarse en los aspectos técnicos de su exégesis y subrayando su pertenencia a la tradición de la exégesis espiritual. El objetivo del teólogo francés fue demostrar que la verdadera novedad de santo Tomás respecto a los comentaristas anteriores no consiste en emplear métodos exegéticos distintos, sino en conceder a la exégesis el lugar diferente dentro del conjunto de su proyecto teológico.

\begin{abstract}
The article analyses the manner in which Henri de Lubac represented saint Thomas of Aquinas in his book on medieval exegesis. The first part is a general study of Exégèse médiévale. The second part is an analysis of the chapter of the book dedicated to saint Thomas and to the scholastic age. The general study of the work previously performed permits to understand why de Lubac presented saint Thomas in a specific way: without focusing on the technical aspects of his exegetical method and with a strong accent put on his appurtenance to the tradition of the spiritual exegesis. His goal was to prove that the originality of Aquinas in regard of the previous commentarists of the Bible doesn't consist in new exegetical methods, but in the different place that the sacred text occupies in his theological reflection.
\end{abstract}


Palabras claves: alegoria; escolástica; exegesis; hermenéutica; Henri de Lubac; Tomás de Aquino.

Keywords: allegory; scholastics; exegesis; hermeneuthics; Henri de Lubac; Thomas of Aquinas.

$\mathrm{C}^{\mathrm{l}}$ propósito de este artículo es estudiar la imagen de Santo Tomás de AquiCno - en cuanto exégeta y teórico de la exégesis - que aparece en la obra Exégèse médiévale de Henri de Lubac. Se trata de una obra publicada entre los años 1959 y 1964 y que consta en más que 1800 páginas, distribuidas en dos partes, de dos volúmenes cada una ${ }^{1}$. Aunque desde su publicación han pasado muchos años, sigue siendo una referencia importante en el estudio de la historia de la exégesis ${ }^{2}$. El subtítulo del libro, "Les quatre sens de l'Écriture", muestra bien que el interés principal del teólogo francés al escribirlo fue el modo de leer e interpretar la Sagrada Escritura, desarrollado a lo largo de la Edad Media, que, detrás de la letra del texto bíblico (sentido literal, primero de los cuatro) buscaba el triple sentido espiritual: la alegoría (sentido doctrinal), la tropología (sentido moral) y la anagogía (sentido escatológico o místico) $)^{3}$. Según testimonia el propio de Lubac, desde que publicó su estudio sobre la exégesis de Orígenes (Histoire et Esprit, 1950), cada vez más se daba cuenta de la importancia capital del esquema de los „cuatro sentidos” en la historia y en la doctrina de la Iglesia. Así, la Exégèse médiévale es fruto de la convicción de que la exégesis espiritual, elaborada en la Edad Media, „comunmente desconocida en la edad moderna”, trae „promesas de renovación” para la teología y la exégesis modernas 4 .

El orden de la exposición de nuestro tema va a ser el siguiente. Primero haremos un breve estudio general de la obra, para averiguar su „arquitectura interna”, es decir, su propósito y su estructura. Segundo, vamos a examinar de cerca los lugares donde de Lubac trata de Santo Tomás de Aquino, y, más generalmente, de la exégesis escolástica. En las conclusiones, vamos a ofrecer una síntesis de la imagen del Aquinate que brota de las páginas de la Exégèse médiévale, e inentaremos sacar a la luz la visión teológico-histórica que está en la base de esta imagen.

1 Las referencias a lo largo del artículo las vamos a hacer de manera siguiente: las abreviaciones: EM1 y EM2 corresponderán a los dos volúmenes de la primera parte, y EM3 y EM4 a los de la segunda.

2 Cf. G.Dahan, Lire la Bible, p. 200; para algunas críticas respecto al valor histórico de la obra de de Lubac, cf. K.L. Hughes, The 'Fourfold sense', pp. 451-453.

3 H.de Lubac, Sur un vieux distique pp. 122-123.

4 H.de Lubac, Mémoire en occassion, p. 84. 


\section{La Exégèse médiévale de Henri de Lubac}

Henri de Lubac afirma en la introducción a su obra que no era su idea escribir una historia de la exégesis de la Edad Media ${ }^{5}$. No se planteaba observar y explicar la lógica de las transformaciones de la manera de leer la Biblia por los medievales, ni le interesaban demasiado los aspectos técnicos de esta actividad. Un pasaje que encontramos ya al principio de la obra resume bien lo que en realidad fue la „exégesis espiritual” que tanto le interesaba:

Porque este «acto completo» que es la antigua exégesis cristiana es una cosa muy grande [...]. Ella define las relaciones de la realidad histórica y de la realidad espiritual, de la sociedad y del individuo, del tiempo y de la eternidad; contiene, como se diría hoy, toda una filosofía de la historia, en conexión con la teología de la Escritura. Ella organiza toda la revelación en torno a un centro concreto, marcado en el espacio y en el tiempo por la Cruz de Cristo. Ella misma constituye una dogmática y una espiritualidad completas, y completamente unificadas. [...]. En una palabra, esta antigua exégesis cristiana es un cosa muy distinta de una forma antigua de la exégesis [...]. Es, bajo uno de sus aspectos esenciales, el antiguo pensamiento cristiano ${ }^{6}$.

De Lubac observa que en su versión clásica (historia, alegoría, tropología y anagogía), el método de los cuatro sentidos fue formulado tarde, cuando ya no tenía mucha importancia en la práctica ${ }^{7}$. No obstante, su estudio está edificado sobre la convicción de que la doctrina de los cuatro sentidos es el fondo común de todos los autores a lo largo de la Edad Media ${ }^{8}$.

Hay que señalar que, aunque la obra se titula Exégèse médiévale, los métodos exegéticos no están en el centro de atención. Si, según la distinción que hace el mismo de Lubac, el término "hermenéutica” se refiere al principio de la interpretación del texto, mientras que el de la „exégesis”, a la práctica ${ }^{9}$, su

5 „Lexamen que nous entreprenons (...) sera beacoup moins une contribution à l'histoire de l'exégèse proprement dite qu'à l'histoire de la théologie, ou plutôt de la pensée et de la spiritualité chrétienne en général", EM1 p. 11; cf. EM1 p. 20.

6 EM1 pp. 16-17.

7 Su codificación tuvo lugar en los comienzos del s.XIII, cf. G.Dahan, Lire la Bible, p. 207.

$8 \quad$ EM1 p. 118.

9 „Les mots d'herméneutique et d'exégèse ont le même sens originel; mais l'usage applique le premier plutôt aux principes d'interprétation, le seconde à la pratique", EM2 p. 662, n.1. 
interés se dirige ciertamente a la primera ${ }^{10}$. Además, el autor subraya varias veces que lo que le interesa no es sólo un principio para leer los textos, sino un principio que organiza todo el universo intelectual y espiritual de los medievales ${ }^{11}$. La „exégesis de los cuatro sentidos” aparece, entonces, como una síntesis orgánica de todas las dimensiones de la vida, de la actividad y del pensamiento cristianos, estructurada alrededor del misterio de Jesucristo ${ }^{12}$.

La convicción de que la exégesis espiritual (llamada también „alegórica”) es la tradición compartida por toda la Edad Media (la escolástica incluida ${ }^{13}$ ) sitúa a de Lubac en las antípodas de varios autores que no veían en el alegorismo medieval más que una etapa del proceso que acabó, felízmente, con el surgimiento de la exégesis científica ${ }^{14}$. Esta es la razón por la que Exégèse médiévale a menudo adquiere un fuerte tono polémico. Los historiadores mencionados más a menudo en este contexto son: C.Spicq y B.Smalley ${ }^{15}$, cuyo argumento de Lubac resume de la manera siguiente: aparte de la exégesis espiritual, en la Edad Media existía e iba ganando cada vez más fuerza una corriente exegética mucho más interesada en la letra del texto bíblico, y, como tal, heredera de San Jerónimo; los autores que pertenecían a ella se independizaban poco a poco de los métodos exegéticos tradicionales y de esta manera protagonizaron un proceso que desembocó en la aparición de la exégesis científica moderna ${ }^{16}$. Según el teólogo francés, esta visión del conjunto de la historia de la exégesis,

10 Cf. „[De Lubac] is claiming that the book explores the intention of the ancient and medieval authors, not their particular practices. In other words, he aims to portray a mentalité, not a method (...). Exégèse Médiévale is the exploration of a theological hermeneutic, not of exegetical technique", K.L. Hughes, The 'Fourfold sense', p. 454.

11 Cf. EM1 pp. 67-68; como observa Robert J. Woźniak, el gran mérito de la obra de de Lubac es poner de manifiesto el vínculo entre la exégesis y el universo mental de los autores medievales, cf. An emerging theology, pp. 416-417.

12 Cf. EM1 pp. 16-17; consideraciones semejantes sobre la exégesis medieval como una „arquitectura”, una manera de organizar el universo y una „lógica de sentido”, cf. O. Boulnois, L'histoire, le corps, l'architecte, pp. 93-100; cf. D. Grumett, Henri de Lubac: Looking for Books to Read the World, pp. 236-249.

13 Cf. Histoire et Esprit, p. 422.

14 Cf. especialmente EM3 pp. 9-14; estas páginas, aunque formalmente pertenecen al primer capítulo, son un tipo de introducción a la segunda parte la obra; cf. también EM3 pp. 199-285.

15 C.Spicq, Esquisse d'une histoire; B.Smalley, The study of the Bible; vale la pena notar que la crítica que de Lubac hace de estos estudios se limita a la vision global que proponen, demasiado simplificada y basada en un prejuicio moderno sobre el progreso. En cambio, en cuanto al análisis de los métodos exegéticos medievales, el teólogo remite a las mismas obras como autoritativas en su materia, cf. EM2 p. 662.

16 EM3 pp. 10-12. 
demasiado „evolucionista” y „finalista”, está basada más en un „mito” que en el examen paciente de los textos ${ }^{17}$. Frente a esta visión, de Lubac no deja de recordar que „el interés por la letra y el gusto predominante por el espíritu no están necesariamente condenados al divorcio; el sentido de la historia y el impulso místico no son de ninguna manera incompatibles"18.

$\mathrm{Al}$ hacer el examen general de Exégèse médiévale, hemos sacado a la luz el doble propósito de la obra: primero, retratar la „exégesis de los cuatro sentidos" como el principio organizador del pensamiento cristiano medieval; segundo, combatir la visión „progresista” de la historia de la exégesis, basada en una valoración negativa de la exégesis espiritual. Este doble propósito se refleja, hasta cierto grado, en la estructura de la obra ${ }^{19}$. La primera parte de la Exégèse médiévale, más sistemática y más teológica, es donde de Lubac se ocupa sobre todo de ofrecer un retrato de la „exégesis de los cuatro sentidos” en cuanto tradición común de la Edad Media. La segunda parte tiene carácter más histórico y más polémico a la vez. En ella, de Lubac analiza a los autores considerados como protagonistas en la "narración del progreso” con el fin de mostrar que la intención de los más grandes exégetas medievales no era tanto superar la tradición, sino sintetizarla con las nuevas exigencias ${ }^{20}$.

Es en la segunda parte de la obra donde se sitúa el capítulo dedicado a la exégesis en la „edad escolástica" ${ }^{21}$. El capítulo consta de cinco subcapítulos, de los que los dos primeros están dedicados casi exclusivamente al Aquinate. Estos dos subcapítulos van a servir de base para nuestra análisis del retrato de santo Tomás que de Lubac dibujó en su obra ${ }^{22}$.

17 EM3 p. 199; cf. EM1 p. 16; el „mito” de que se trata aquí es, según parece, el mito moderno del progreso absoluto en el pensamiento humano, al que de Lubac no deja de combatir en las más importantes de sus obras, p.ej. H.de Lubac, Le mystère du surnaturel, pp. 19-40; cf. F.Bertoldi, De Lubac. Cristianesimo e modernità, pp. 83-90.

18 EM2 p. 665; cf. EM2 p. 663.

19 Cf. las respectivas introducciones a las dos partes de la obra, EM1 pp. 11-39; EM3 pp. 9-14.

20 Son de primera importancia los capítulos dedicados a Hugo de san Víctor y a su escuela, cf. EM3 pp. 287-435.

21 „L'age scolastique”, pp. EM4 pp. 263-367.

22 El capítulo mencionado no es el único lugar donde aparece el nombre del Aquinate. Aparte de él, a lo largo la obra aparecen 45 menciones ocasionales, cf. índices de personas: EM2 p. 709; EM4 p. 549. 


\section{Santo Tomás de Aquino en la Exégèse médiévale.}

El primero de los dos subcapítulos que vamos a tratar aquí está dedicado a la exégesis en la obra de los „dos grandes maestros”: san Buenaventura y Santo Tomás $^{23}$. La parte dedicada a san Buenaventura es breve ${ }^{24}$, así que no cabe duda de que el interés principal de de Lubac se dirige sobre todo al segundo de los dos maestros. Tomando como punto de referencia el simbolismo sobreabundante de la exégesis bonaventuriana, de Lubac observa que „el estilo del Doctor angélico es otro”, „sus exposiciones son más sobrias”, pero, aparte de algunos matices, „su doctrina es la misma” 25 . Por consiguiente, empieza a tratar los lugares de la obra tomasiana donde esta doctrina queda formulada. Entre estos, destacan tres principales: el Quodlibet VII, el Comentario de la Carta a los Gálatas y la primera Quaestio de la Prima Pars.

El lector pronto se da cuenta de que el exámen de las tres obras mencionadas sirve a de Lubac sobre todo para demostrar la pertenencia de Santo Tomás a la tradición de la „exégesis espiritual”. Primero, el autor resume los dos artículos de Quodlibet VII; en el 14 el santo plantea la cuestión de si hay en la Escritura dos sentidos, el literal y el figurativo; en el 15 la cuestión es la supuesta existencia de los 4 sentidos en la Sagrada Escritura, para concluir que a las dos preguntas el Aquinate responde afirmativamente ${ }^{26}$. De Lubac cita un extracto de la cuestión 15, que es una síntesis clara y ordenada del „esquema de los cuatro sentidos"27. En cuanto al Comentario a Gálatas, el jesuita francés se fija en lo que se dice allí respecto al texto que tiene importancia capital para la configuración de la exégesis alegórica: la afirmación paulina de que la historia de Sara y Hagar „tiene también sentido alegórico” 28 . El teólogo hace notar que santo Tomás apunta a una dualidad de significados de la palabra "alegoría”: el término en su aplicación general se refiere a todo lo que no es la "letra” del texto, mientras que en otras ocasiones designa el segundo de los cuatro sentidos bíblicos ${ }^{29}$. Al final, de Lubac examina el artículo 10 de la primera cuestión de la Prima Pars, donde se discute la existencia de la „pluralidad de sentidos

\footnotetext{
23 „Les deux grands maîtres”, EM4 pp. 263-285.

24 Cf. EM pp. 264-272.

25 EM4 p. 272.

$26 \quad$ EM4 pp. 273-274; cf. G.Dahan, Lire la Bible, p. 215.

27 EM4 p. 273.

28 Super Gal., cap. 4 1.7; EM4 p. 274; el versículo bíblico es: Ga 4,24.

$29 \quad$ EM4 p. 274.
} 
bajo la letra única" de la Sagrada Escritura ${ }^{30}$. Su observación inicial es que dicho artículo no añade nada esencial a lo expuesto en los dos textos anteriores: apoyándose en la autoridad de San Gregorio Magno, santo Tomás afirma que bajo la letra del texto bíblico, hay un sentido espiritual, y éste se divide en tres: alegórico, tropológico y anagógico, todo según el „viejo esquema” común de toda la Edad Media ${ }^{31}$.

No obstante, aparte de afirmar la pertenencia del Aquinate a la tradición, de Lubac señala tres rasgos particulares que se puede encontrar en la primera quaestio de la Summa. Primero, se fija en la insistencia de santo Tomás en que el argumento teológico sólo puede basarse en la letra del texto, y nunca en la alegoría $^{32}$. El teólogo francés observa que se trata de una necesidad urgente en el contexto del cambio del método que experimentó la teología, pero es difícil ver aquí una verdadera novedad: la misma idea se encontraba ya en san Agustín, Rábano Mauro y Pseudo-Dionisio ${ }^{33}$. El segundo punto señalado es la distinción neta que hace santo Tomás entre la metáfora - que pertenece al sentido literal -, y la alegoría propiamente dicha ${ }^{34}$. En este caso, de Lubac reconoce el mérito que tiene el Aquinate, pero lo limita a la solución de una confusión meramente terminológica, puesto que, según él, los exégetas medievales a menudo utilizaban los dos términos de manera confusa, pero nunca confundían las dos realidades a las que dichos términos se referían ${ }^{35}$.

Como tercer rasgo particular destacado por el teólogo francés aparece la idea de una „cierta pluralidad del mismo sentido literario” admitida por Tomás ${ }^{36}$. Aquí de Lubac se detiene más; primero relata el débate que se desarrolló en torno a esta afirmación del Doctor Angélico, con las dos opiniones confrontadas: unos que querían interpretar el versículo de la Suma en el sentido más obvio (así, se pronunciaban a favor de la pluralidad de sentidos literarios), y otros que subrayaban la falta de claridad del texto tomasiano y sostenían que la pluralidad se refiere, en realidad, al conjunto de sentidos de la Biblia, no a la

\footnotetext{
30 S.Th.I, q1, a10.

31 S.Th.I, q1, a10., citado en EM4 p. 276.

32 S.Th.I, q1, a10, ad1., citado en EM4 p. 279; cf. Histoire et Esprit, p. 423; cf. „The fundamental prerequisite for the understanding of exegetical language of Thomas Aquinas is the priority of the literal sense over other meanings of text identified by him", P. Roszak, Between Dialectics and Metaphor, 511.

$33 \quad$ EM4 p. 277.

34 EM4 pp. 277-278; cf. P. Roszak, Between Dialectics and Metaphor, p. 514

$35 \quad$ EM4 p. 278.

36 Como otros lugares donde aparece la misma idea, de Lubac señala dos: In 2 Sent., d12, q1, a2, ad 7; De Potentia, q4, a1.
} 
sola littera ${ }^{37}$. Después, analiza las diversas traducciones al francés del versículo en cuestión ${ }^{38}$. Finalmente, propone su propia solución de la controversia, empleando el fragmento de De Potentia para aclarar el texto difícil de la Suma. Esta solución se puede resumir en tres puntos. Primero, el sentido de la afirmación de santo Tomás es sobre todo pragmático: permite „mantener por igual las opiniones diversas de los intérpretes autorizados, de encontrar en la Escritura todo pensamiento justamente concebido y, finalmente, defender con más eficacia la Palabra de Dios" ${ }^{39}$. Segundo, dicha afirmación autoriza a elegir entre varios sentidos más bien que a sumarlos. Tercero, se establece en ella una gradación de la „literalidad” o „autenticidad” de estos sentidos: desde el sentido directamente concebido por el autor humano del libro, pasando por el solamente previsto por este autor, hasta el sentido no previsto por el autor, pero sí por el Espíritu Santo. De Lubac concluye que, aunque sólo en los primeros dos casos se puede hablar de los „sentidos del autor”, y en el tercer caso, solamente de "sentidos adaptados", todos tres son considerados verdaderos sentidos de la Escritura, sentidos contenidos en ella ${ }^{40}$. Extrañamente, el largo análisis que el teólogo hace de la cuestión de la "pluralidad de sentidos literarios” no viene acompañado por ninguna observación concluyente, salvo la afirmación de la perfecta coincidencia entre las consideraciones al respecto que se encuentran en la Suma y en las otras obras del Aquinate.

El segundo subcapítulo, titulado „La «nouveauté» de saint Thomas”, comienza con una afirmación de carácter recapitulatorio: tanto en los tres textos esenciales de santo Tomás, como en otros en los que aparece el tema de la Escritura, a pesar de algunas diferencias de matices, el Aquinate se muestra como testigo de la tradición exegética compartida por toda la Edad Media ${ }^{41}$. No es una casualidad que la palabra „novedad” en el título del subcapítulo aparezca entre paréntesis: a lo largo de él, de Lubac entra en polémica con los que veían en el Doctor Angélico uno de los protagonistas del proceso de la sustitución de la vieja exégesis alegórica por la nueva, científica ${ }^{42}$. Su propio juicio sobre santo Tomás lo resumen estas palabras:

37 En el primer grupo, de Lubac entre varios autores enumera a A.-D.Sertillanges y E.Gilson, en el segundo, a A.Gardeil y C.Spicq, cf. EM4 pp. 279-282.

38 Es decir, S.Th.I, q1, a10 co, In 2 Sent., d12, q1, a2, ad 7 y De Potentia, q4, a1.

39 EM4 p. 284.

$40 \quad$ EM4 pp. 284-285.

$41 \quad$ EM4 p. 285.

42 Cf. EM4 pp. 286-295; entre los méritos de la exégesis tomasiana se solía enumerar: el rechazo del alegorismo exagerado, el hecho de "poner el sentido espiritual en las cosas”, la distinción clara entre alegoría y metáfora; de Lubac procura demostrar que ninguna de estas 
Sin querer innovar en nada, se contentó con expresar en términos sobrios y netos [...] una doctrina de doce siglos, en la que se afirma la originalidad de la alegoría cristiana. Bella ilustración de esa verdad, según que un genio es, en el mejor sentido de la palabra, un simplificador. Gracias a sus cualidades ordinarias de robusta simplicidad, de adecuación y de precisión, santo Tomás resume la enseñanza común con éxito ${ }^{43}$.

Estas palabras se refieren, en primer lugar, a lo que santo Tomás dijo sobre sus principios exegéticos. En cuanto a la propia práctica exegética del Aquinate, de Lubac no nos ofrece muchos datos. En algunos lugares, señala la presencia de un nuevo acercamiento al texto bíblico en los comentarios escritos por el santo - sobre todo en el Comentario al Libro de Job, centrado en el sentido literal del libro comentado y lleno de la argumentación dialéctica. Sorprendentemente, lejos de subrayar la importancia de estos rasgos aparentemente novedosos, el teólogo procura disminuirla: por un lado, se contenta con la afirmación del Aquinate de que su fijarse en la letra del texto no es por despreciar al método alegórico, sino por no poder añadir ya nada al comentario alegórico hecho por san Gregorio el Grande ${ }^{44}$. Por el otro, sostiene que, detrás de su „armadura dialéctica”, el comentario que hace Tomás sigue siendo lo que siempre ha sido: la explicación de la Escritura por la propia Escritura y según los Padres ${ }^{45}$. En resumen, el teólogo afirma que santo Tomás no sólo habla de la „exégesis de los cuatro sentidos”, sino también la practica ${ }^{46}$. No obstante, hay en el Aquinate un rasgo distinctivo: el carácter moderado y sobrio del simbolismo que se encuentra en sus comentarios bíblicos. Aquí de Lubac ve un signo de que el Doctor Angélico tenía otra misión por cumplir. En vez de enriquecer „el vasto tesoro del simbolismo medieval”, lo suyo era elaborar „la gran edificación doctrinal de la Suma"47.

„innovaciones” es una novedad en sentido absoluto, puesto que puede contar con varios antecedentes en la historia.

$43 \quad$ EM4 p. 286.

$44 \quad$ EM4 p. 298.

$45 \quad$ EM4 p. 301; curiosamente, de Lubac parece no notar la diferencia entre los antiguos exégetas, que llegaban más allá del sentido literal mediante el movimiento de la „anagogía espiritual”, y el Aquinate, que procura alcanzarlo mediante el razonamiento metafísico; sobre la metafísica como el „sistema nervioso” de la exégesis tomasiana, 'f. P. Roszak, Exégesis y metafísica, pp. 313-315.

46 EM4 p. 296; a esta observación siguen algunos ejemplos, cf. EM4 p. 299; una opinión distinta sobre la presencia de la exégesis espiritual en la práctica del Aquinate, cf. G.Dahan, Lire la Bible, p. 217.

$47 \quad$ EM4 p. 299. 
Esta observación nos lleva hacia lo que es, según de Lubac, la verdadera novedad de santo Tomás. Ésta radica no tanto en adoptar los métodos exegéticos nuevos, sino en conceder una finalidad distinta a la exégesis. En las épocas anteriores, „la teología y la explicación de la Sagrada Escritura no eran sino una misma cosa" ${ }^{48}$. Así, un comentario bíblico escrito según el esquema de la „exégesis espiritual” era un punto de llegada de la reflexión creyente. En la escolástica, en cambio, los comentarios empiezan a servir como fuente de datos que luego quedan incorporados en „sistematizaciones de un carácter distinto" 49 . El punto de llegada ya no es el comentario, sino la Suma. Si en la primera cuestión de Prima Pars el Aquinate todavía parece confundir la sacra doctrina con la sacra Scriptura, a partir de la segunda todo queda claro: a pesar de subrayar al principio la importancia de la exégesis „según las concepciones tradicionales", el santo escribió su obra siguiendo principios distintos. Como afirma de Lubac, „detrás de una portada románica, el arquitecto ha construido una inmensa nave gótica" ${ }^{50}$.

De Lubac sostiene que la obra de santo Tomás es el lugar donde el nuevo tipo de la sistematización en teología alcanzó su perfección. Antes de pasar a las conclusiones, vamos a subrayar, siguiendo al teólogo francés, dos rasgos que diferencian a este nuevo método (es decir, el escolástico) del pensamiento tradicional (es decir, el plasmado sobre el modelo de los „cuatro sentidos”).

El primer rasgo que parece tener una importancia particular es el cambio de sentido de la "anagogía”, tradicionalmente identificada con el cuarto sentido de la Escritura, vinculado con la escatología y con el fin último del hombre. En el esquema tradicional, en el que destaca la unidad estrecha de los cuatro sentidos entre sí, la anagogía aparecía como la plenitud de los demás sentidos. En cuanto plenitud a que tendía el sentido alegórico (dotrinal), señalaba que la doctrina de la Iglesia tiene una estructura dinámica, y, en cuanto a su asimilación y plena comprensión, está en camino hacia el pleno cumplimiento en el eschaton. En cuanto plenitud de la tropología (sentido moral), recordaba que toda la reflexión y toda la actividad del creyente no tiene su razón de ser en sí, sino que sirve para que el hombre pueda alcanzar su fin último, la unión perfecta con Dios. El sentido de la anagogía, según de Lubac, cambia en la teología escolástica. En las Sumas, los tres sentidos espirituales se independizan del comentario bíblico para constituirse en un cuerpo de doctrina que sigue ya otros

$48 \quad$ EM1 p. 59.

49 EM4 p. 301.

$50 \quad$ EM4 p. 301; sobre el lugar de la Sagrada Escritura en la teología de santo Tomás, cf. R.J.Woźniak, An emerging theology, pp. 421-423. 
métodos ${ }^{51}$. La anagogía que se encuentra dentro de este cuerpo doctrinal, cambia de sentido. Para Buenaventura, el primer objeto de la anagogía es el estudio de la Trinidad; para Alejandro de Hales, - la fe en la divinidad; todo esto sin una relación particular con el fin último del hombre, es decir, con la escatología o con la contemplación ${ }^{52}$. El misterio cristiano ya no es considerado como un designio dinámico que se realiza en el tiempo y que tiende hacia el eschaton, sino más bien como una obra de Sabiduría divina, estática y perfectamente desarrollada ${ }^{53}$.

El segundo rasgo es la pérdida de la unidad orgánica entre los varios elementos de la síntesis cristiana. En la teología plasmada sobre los „cuatro sentidos”, se veía con claridad los vínculos que unían el texto bíblico con la doctrina, la doctrina con la dimensión moral y existencial del misterio, la doctrina y la existencia con la esperanza escatológica. El paso a la estructura de las cuestiones y de las Sumas supuso un "desmembramiento" de la síntesis del misterio cristiano $^{54}$. La exégesis, la doctrina, la moral y la escatología siguien siendo elementos importantes de la síntesis cristiana, pero ya no es tan claro cómo se relacionan entre sí y cómo se unen.

La actitud de de Lubac ante estos cambios, efectúados en la teología y provocados por la actitud distinta respecto al texto bíblico, es compleja. Por un lado, varias veces afirma que en tiempos de santo Tomás, cuando la doctrina de los cuatro sentidos quedó codificada, era ya una realidad muerta. Su existencia era una mera supervivencia. Perfectamente objetivizada, ya hacía tiempo que no constituía el „alma” del pensamiento medieval. Los escolásticos, aunque no sentían ninguna necesidad de cambiar la fórmula tradicional, la empleaban de manera puramente mecánica ${ }^{55}$. A la vez, el teólogo reconoce que el cambio de método y la división entre la exégesis y la doctrina „era una condición indispensable del progreso tanto de una como de otra" ${ }^{56}$ y que tanto la exégesis, como la teología y la espiritualidad „han sacado un verdadero provecho de su liberación recíproca" 57 .

$51 \quad$ EM4 p. 300; p. 310.

52 EM4 p. 300.

53 EM3 p. 421.

54 Sobre este „desmembramiento" que ocurría ya en tiempos de Hugo de San Víctor, cf. EM3 pp. 418-435.

$55 \quad$ EM4 pp. 309-310.

56 EM3 p. 423.

57 EM3 pp. 423-424. 
No obstante, los autores más valorados por de Lubac son los que, dentro de las nuevas condiciones, lograron hacer una síntesis del misterio cristiano. En esta línea se sitúan, según él, los grandes maestros de la escolástica:

No olvidemos [...] que, en su cumbre - que duró, desgraciadamente, demasiado poco - la gran Escolástica logró, a su manera, una verdadera síntesis de las tres disciplinas. Los maestros de la doctrina eran a la vez los maestros en la Sagrada Escritura, y su obra está todavía llena de la savia espiritual. Si en la Suma de santo Tomás hay más de historia que en otros teólogos posteriores, es porque en ella hay también más de la Escritura ${ }^{58}$.

Así, el Aquinate, aunque pertenece plenamente a la nueva mentalidad teológica, más centrada en el razonamiento y la sistematización que en la lectura espiritual, no se sitúa en la línea de la escisión, sino en la de la síntesis. El reconocimiento que de Lubac hace de este rasgo del autor de la Suma no debe hacernos, sin embargo, olvidar que su ideal de la síntesis era distinto. Como observa J.Guillet, para el jesuita francés la cumbre de la Edad Media no fue la escolástica con sus Sumas y sus universidades, sino la teología monástica del siglo XII, edad de Cluny y de Vézelay, de Guillermo de Saint-Thierry y de San Bernardo $^{59}$. Aunque reconocía los logros de un santo Tomás o de un san Buenaventura, su propuesta de renovación de la teología más tenía que ver con las síntesis basadas en los „cuatro sentidos” que en las sistematizaciones propias de la escolástica. En los tiempos en que escribía su gran obra, la necesidad más urgente que veía no era tanto la sistematización de la doctrina, cuanto la recuperación de la unidad perdida entre las varias dimensiones del misterio.

\section{Conclusión}

Aquí vamos a resumir las principales ideas que han salido a la luz a lo largo de nuestro estudio.

1. En su obra, de Lubac no se detiene mucho en el análisis de los métodos exegéticos del Aquinate. Para un lector interesado en esta materia, la Exégèse médiévale no parece tener mucho valor.

2. El estudio de la exégesis de santo Tomás en la Exégèse médiévale depende del doble propósito general de la obra: la demostración de la unidad de la tradición exegética a lo largo de la Edad Media y la polémica contra los

$58 \quad$ EM4 p. 425.

59 J. Guillet, Le sens de l'Écriture, p. 360; cf. EM3 pp. 426-427. 
historiadores de la exégesis que veían en ella un proceso de sustitución de las fantasías alegoristas por la exégesis verdadera (científica). En las páginas dedicadas al santo, de Lubac se empeña en mostrar el carácter perfectamente tradicional de su actitud en la exégesis; el Aquinate, según él, no quería cambiar nada en la manera de interpretar la Sagrada Escritura y afirmaba, junto con toda la tradición, el valor perenne de la exégesis „según los cuatro sentidos”.

3. El teólogo francés ve la verdadera innovación de santo Tomás y sus contemporáneos no tanto en el cambio de los principios exegéticos, cuanto en el distinto papel que la exégesis adquiere dentro del conjunto de la reflexión teológica: en vez de ser el producto final de dicha reflexión, pasa a ser una base de datos revelados para la elaboración de las Sumas. Aunque los comentarios en la edad escolástica se siguen escribiendo según el principio de los „cuatro sentidos”, este principio ya no es el que organiza las sistematizaciones de la doctrina.

4. El nuevo tipo de sistematización del misterio cristiano tiene tanto sus ventajas, como sus inconvenientes. Entre los inconvenientes, de Lubac subraya la incapacidad de mostrar el carácter orgánico de la síntesis cristiana y la pérdida de la dimensión escatológica de éste. Ambas deficiencias se deben al abandono del equema de los „cuatro sentidos” como principio hermenéutico en la reflexión teológica.

5. Santo Tomás aparece en la obra de de Lubac como un personaje que se escapa de las generalizaciones fáciles. Uno de los innovadores en la teología, en cuanto a la exégesis se muestra fiel a la vieja tradición. Miembro de un movimiento intelectual cuyos métodos de sistematización contribuían a la división entre varias disciplinas teológicas, se esfuerza en la elaboración de una síntesis donde puedan coexistir la exégesis, el razonamiento y la dimensión espiritual-existencial del misterio cristiano.

6. Parece que de Lubac quiere ver en el Aquinate (y también en san Buenaventura) no tanto un autor ejemplar para la escolástica, cuanto una excepción, y esto especialmente gracias a la importancia que en su pensamiento tiene la Sagrada Escritura y gracias a su búsqueda de la síntesis equilibrada entre la exégesis, la teología y la espiritualidad. No obstante, el jesuita francés ve la fuente de inspiración para la renovación de la teología contemporánea sobre todo en los autores anteriores, cuyo pensamiento estaba moldeado según el principio de los „cuatro sentidos”. 


\section{Bibliografía}

Bertoldi, F., De Lubac. Cristianesimo e modernità, Bologna 1994.

Boulnois, O., L’histoire, le corps, l'architecte. L'exégèse médiévale et l'herméneutique, en: Comprendre et interpréter. Le paradigme herméneutique de la raison, (VV.AA.), Paris 1993, 83-100.

Dahan, G., Lire la Bible au Moyen Âge. Essais d’herméneutique médiévale, Genève 2009.

Grumett, D., Henri de Lubac: Looking for Books to Read the World, en: Ressourcement. A movement for Renewal in Twentieth-Century Catholic Theology, G. Flynn, P.D.Murray (ed.), Oxford 2012, 236-249.

Guillet, J., Le sens de l'Écriture. Exégèses d'autrefois, recherches d'aujourd'hui, RSR 80/3 (1992), 359-372.

Hughes, K.L., The 'Fourfold sense: de Lubac, Blondel and Contemporary Theology, HeyJ XLII (2001), 451-462.

Lubac de, H., Exégèse médiévale, t.I-IV, Paris 1959-1964.

Lubac de, H., Histoire et Esprit, Paris 1950.

Lubac de, H., Mémoire sur l'occasion de mes écrits, Paris 2006.

Lubac de, H., Paradoxes, Paris 1999.

Lubac de, H., Sur un vieux distique. La doctrine du «quadruple sens», en: Théologies d'Occasion, Paris 1984, 117-136.

Roszak, P., Between Dialectics and Metaphor: Dynamics of the Exegetical Practice of Thomas Aquinas, Angelicum 90 (2013) 507-534.

Roszak, P., Exégesis y metafísica. En torno a la hermenéutica bíblica de Tomás de Aquino, Salmanticensis 61 (2014) 301-323.

Smalley, B., The Study of the Bible in the Middle Age, Indiana 1964.

Spicq, C., Esquisse d'une histoire de l'exégèse latine au moyen age, Paris 1944.

Woźniak R.J., An Emerging Theology Between Scripture and Metaphysics: Bonaventure, Aquinas and the Scriptural Foundation of Medieval Theology, en: Reading Sacred Scripture with Thomas Aquinas, P. Roszak, J. Vijgen (eds.), Brepols 2015, 415-434.

Todas citas y referencias a las obras de santo Tomás según http://www.corpusthomisticum.org 